(C) This manuscript version is made available under the CC-BY-NC-ND 4.0 license https://creativecommons.org/licenses/by-nc-nd/4.0/

http://doi.org/10.1016/J.JPAINSYMMAN.2019.12.338 


\section{Providers' Perspectives on Prescribing Long-term Opioid Therapy in Cancer: Qualitative Findings form the United States and Australia}

Soraya Fereydooni, BS, Stanford University. Tim Luckett, PhD, University of Technology Sydney. Jane Phillips, PhD RN, University of Technology Sydney. Karl Lorenz, MD MSHS, VA Palo Alto Health Care System. Karleen Giannitrapani, PhD, VA Health Services Research \& Development and Stanford University.

Objectives

- Understand providers' perspectives on factors impacting opioid prescription in cancer.

- Illustrate the differences and similarities in diverse cultural and political contexts.

Importance. To understand how cultural and political difference ca impact perspectives on opioid therapy in cancer.

Objective(s). 1. To understand providers perspectives on factors impacting opioid prescription in cancer. 2. To illustrate the differences and similarities in diverse cultural and political contexts.

Method(s). Using similar but contextually tailored semi-structured interview guides, we conducted two separate qualitative studies in the U.S. and Australia. We captured the perspectives of 43 providers: 10 general practitioners (GP) and 10 oncology providers (ONC) in the US. Department of Veterans Affairs, 20 GPs and 3 ONCs in Australia. Qualitative content analysis method was used in each study and then the emerging themes were compared across studies.

Results. 1) Patient characteristics impacting decision: Providers in both contexts cited patient prognosis, diversion by patients' family (It's a very common story, obtaining opioids from family members), goals for pain management, important contextual factors (e.g. history of substance misuse, mental health), the cause, type and quality of pain as factors important in assessment for opioid therapy 2) Barriers to shared decision making: Both specified limited clinical time and inadequate communication between providers. 3) Facilitators to shared decision making: Both mentioned having an established therapeutic relationship with the patient. 4) The title cancer: Both quoted "cancer" makes them "a little more liberal with opiate use." Australian GPs specifically mentioned that providers might "have a soft heart for the cancer patients" and are blinded by this title. 5) Use of alternative prescription: Australian providers mentioned opioid alternatives are lacking and its simpler "to write doses of Oxycontin than to get people involved in multi-disciplinary team." Only American prescribers mentioned burdens associated with maintaining patients on opioids.

Conclusion(s). Factors were largely similar in both contexts with some notable contextual differences. Impact. This qualitative study looks at two international contexts; the differences highlight the importance of further international comparisons regarding best practices and policies. 